\title{
Zur Kenntnis des Tyrosinabbaus in der künstlich durch- bluteten Leber.
}

\author{
Von \\ Dr. Otto Neubauer und Dr. Walter GroB. \\ Mit einer Abbildung im Text. \\ (Aus der II. medizinischen Klinik in München.) \\ (Der Redaktion zugegangen am 11. Juni 1910.)
}

Die Untersuchungen der letzten Jahre haben gelehrt, daß die Aminosäuren der Eiweißkörper im Organismus über die Stufe der um 1 C-Atom ärmeren Fettsäuren abgebaut werden. ${ }^{1}$ ) Als erster Schritt bei diesem Abbau ist mit großer Wahrscheinlichkeit die Abspaltung des Stickstoffs in Form von Ammoniak anzusehen (Desaminierung). Während man bisher als Produkt dieser Desaminierung die der Aminosäure entsprechende Alkoholsäure (Oxysäure) betrachtete, hat der eine von uns die Anschauung vertreten, daß die Desaminierung der Aminosäuren im Organismus zum Auftreten der entsprechenden Ketonsäuren führe. ${ }^{2}$ ) Diese Ansicht stützte sich unter anderem auf Erfahrungen, welche sich bei der Verabreichung verschiedener Abkömmlinge des Tyrosins bei einem an Alkaptonurie leidenden Manne ergaben: während die dem Tyrosin entsprechende Ketonsäure, die p- Oxyphenylbrenztraubensäure

$$
\mathrm{HO} \cdot \mathrm{C}_{6} \mathrm{H}_{4}-\mathrm{CH}_{2}-\mathrm{CO}-\mathrm{COOH}
$$

ebenso wie Tyrosin selbst eine Vermehrung der Alkaptonausscheidung bewirkte, war die entsprechende Alkoholsäure, die $p$-Oxyphenyl- $\alpha$-milchsäure

$$
\mathrm{HO} \cdot \mathrm{C}_{6} \mathrm{H}_{4}-\mathrm{CH}_{2}-\mathrm{CHOH}-\mathrm{COOH}
$$

ohne jeden Einfluß. Daraus konnte geschlossen werden, daß

1) Literatur s. bei Otto Neubauer, Deutsch. Arch. f. klin. Med., Bd. XCV, S. 220 (1909).

2) Ebenda, S. 241. 
die Alkoholsäure als Zwischenprodukt beim Tyrosinabbau nicht in Betracht kommt, wohl aber die Ketonsäure. Dieser Schluß gilt zunächst nur für den Abbau des Tyrosins zur Homogentisinsäure, wie er beim Alkaptonuriker als sicher erwiesen gelten kann; eine Übertragung auf den Tyrosinabbau im normalen Organismus erscheint nur in dem Falle begründet, daß die Stoffwechselstörung bei der Alkaptonurie als eine einfache $\mathrm{Hem}$ mung des normalen Stoffwechsels aufgefaßt werden darf. Wenn sich nun auch für diese Auffassung von dem Wesen der Alkaptonurie eine Reihe von guten Gründen anführen lassen, so ist es doch bisher noch nicht möglich gewesen, absolut sichere Beweise für sie beizubringen. Es war daher wünschenswert, die in den Untersuchungen beim Alkaptonuriker gewonnene Ansicht, daß nicht die p-Oxyphenylmilchsäure, wahrscheinlich aber die p-Oxyphenylbrenztraubensäure als Desaminierungsprodukt des Tyrosins entsteht, auf einem anderen, von der Alkaptonurie ganz unabhängigen Weg zu prüfen.

Das wurde ermöglicht durch die von Embden begründete Methode der Untersuchung der Acetonbildung in überlebend gehaltenen Organen. Embden und seine Mitarbeiter ${ }^{1}$ ) haben gezeigt, daß die künstlich (mit Rinderblut) durchblutete Hundeleber geringe Mengen von Aceton (resp. Acetessigsäure) bildet, und daß diese Acetonbildung durch Zusatz bestimmter Substanzen, unter anderen auch der aromatischen Aminosäuren Phenylalanin und Tyrosin, so bedeutend gesteigert werden kann, daß man zu dem Schluß berechtigt ist, daß diese Substanzen von der Leber zu Aceton (resp. Acetessigsäure) abgebaut werden. Es ist nun klar, daß alle Zwischenprodukte, die bei diesem Abbau der aromatischen Aminosäuren auftreten, im Durchblutungsversuch ebenfalls eine Acetonvermehrung bewirken müssen, während anderseits Substanzen, deren Zusatz zu keiner vermehrten Acetonbildung führt, bei der Frage nach den intermediären Produkten beim Abbau der aromatischen Aminosäuren nicht weiter in Frage kommen.

1) Almagia und Embden, Hofmeisters Beitr., Bd. VI, S. 59 (1904). - Embden und Kalberlah, ebenda, Bd. VIII, S. 1 (1906). Embden, Salomon und Schmidt, ebenda, S. 9. 
Von dieser Überlegung ausgehend haben Embden, Salomon und Schmid $t^{1}$ ) Durchblutungsversuche auch mit Homogentisinsäure angestellt; sie erhielten eine beträchtliche Steigerung der Acetonbildung; diese Tatsache ist geeignet, die Vorstellung, daß die Homogentisinsäure auch beim Normalen als intermediäres Abbauprodukt der aromatischen Aminosäuren entsteht, wesentlich zu stützen.

Embden, Salomon und Schmidt haben ferner ihre Versuche auch auf eine Substanz ausgedehnt, welche - da sie beim Alkaptonuriker nach den Untersuchungen von $\mathrm{Neu}-$ bauer und $\mathrm{Falta}^{2}$ ) in Alkapton übergeht, vom normalen Tier vollständig verbrannt wird (Knoop $\left.{ }^{3}\right)$ ) - als Zwischenprodukt beim Übergang des Phenylalanins in Homogentisinsäure aufgefaßt werden kann, auf die Phenyl- $\alpha$-Milchsäure

$$
\mathrm{C}_{6} \mathrm{H}_{5}-\mathrm{CH}_{2}-\mathrm{CHOH}-\mathrm{COOH} \text {. }
$$

Da auch diese Substanz, im Gegensatz zu anderen ähnlichen aromatischen Säuren (Phenylpropionsäure, Phenyl- $\beta$-Milchsäure) im Durchblutungsversuch Aceton lieferte, so ergab sich also ein vollkommener Parallelismus mit den Resultaten von Neubauer und Falta und denen von Knoop: diejenigen aromatischen Säuren, die beim Alkaptonuriker eine Alkaptonvermehrung bewirken, sind im normalen Organismus glatt verbrennbar und liefern bei den Durchblutungsversuchen als Endprodukt Aceton.

Nachdem nun die eingangs erwähnten Versuche des einen von uns gelehrt hatten, daß die entsprechenden Abkömmlinge des Tyrosins sich beim Alkaptonuriker etwas anders verhalten wie die des Phenylalanins, indem nur die Ketonsäure, nicht aber die Alkoholsäure eine Vermehrung der Alkaptonkörper bewirkt, erschien es von Interesse, auch diese Säuren auf ihre Fähigkeit zu prüfen, beim Durchleiten durch die überlebende Leber in Aceton überzugehen.

1) Embden und Schmidt, a. a. O. S. 33.

2) Neubauer und Falta, Diese Zeitschrift, Bd. XLII, S. 96 (1904).

$\left.{ }^{3}\right)$ Knoop, Der Abbau d. aromat. Fettsäuren im Tierkörper, Hab.Schrift, Freiburg 1904. 
Die Methodik unserer Versuche schloß sich im wesentlichen der Embdens und seiner Mitarbeiter an.

Auch wir stellten die Untersuchungen an Hundelebern an. Zur Durchblutung verwendeten wir aber nicht Rinderblut, sondern Hundeblut, und zwar regelmäßig das durch Schlagen defibrinierte und mit Ringerlösung verdünnte Blut desselben Tieres, von dem die Leber stammte; wir glauben so unter natürlicheren Bedingungen gearbeitet zu haben, als bei Verwendung eines artfremden Blutes. Um recht gut vergleichbare Verhältnisse zu haben, haben wir ferner darauf geachtet, daß das Verhältnis des Lebergewichtes zur Menge der Durchströmungsflüssigkeit in allen Fällen das gleiche war: wir nahmen immer eine dem Gewichte der Leber gleiche Blutmenge und verdünnten sie mit Ringerlösung und mit der Lösung der $z u$ prüfenden Substanz genau auf das 3 fache.

Der Durchblutungsapparat, dessen wir uns bedienten, wich im Prinzip nicht wesentlich von den Apparaten ab, wie sie von anderen Autoren für ähnliche $Z$ wecke angegeben worden sind; im einzelnen haben wir allerdings eine Reihe von Veränderungen und Modifikationen vorgenommen, die sich uns beim praktischen Gebrauch gut bewährt haben, und welche es rechtfertigen dürften, wenn wir unser Modell genauer beschreiben.

Als Triebkraft für den Apparat stand uns ein auf einem etwa $40 \mathrm{~cm}$ hohen Tischchen montierter Elektromotor (1/6 PS) zur Verfügung; er drehte eine Achse, an der eine exzentrische Scheibe E derart befestigt war, daß der Grad ihrer Exzentrizität mittels eines Schlitzes und einer Schraube verschieden eingestellt werden konnte. Die exzentrische Scheibe drückt bei ihren Umdrehungen rhythmisch auf eine Klappe $\mathrm{K}$ (ein viereckiges, an der einen Kante mittels eines Scharniers auf der Tischplatte beweglich angebrachtes Brettchen). Unter dieser Klappe liegt das «Herz» des Apparates, ein einfacher, überall käuflicher Gummi-Klysopomp (H) mit 2 Bleiventilen, ${ }^{1}$ ) die so

1) Die Vorteile der Gummi-Klysopompe für diesen Zweck hat der eine von uns bereits vor Jahren in einer im Prager pharmakologischen Institut unter Leitung von Herrn Prof. J. Pohl durchgeführten Versuchsreihe erprobt. 


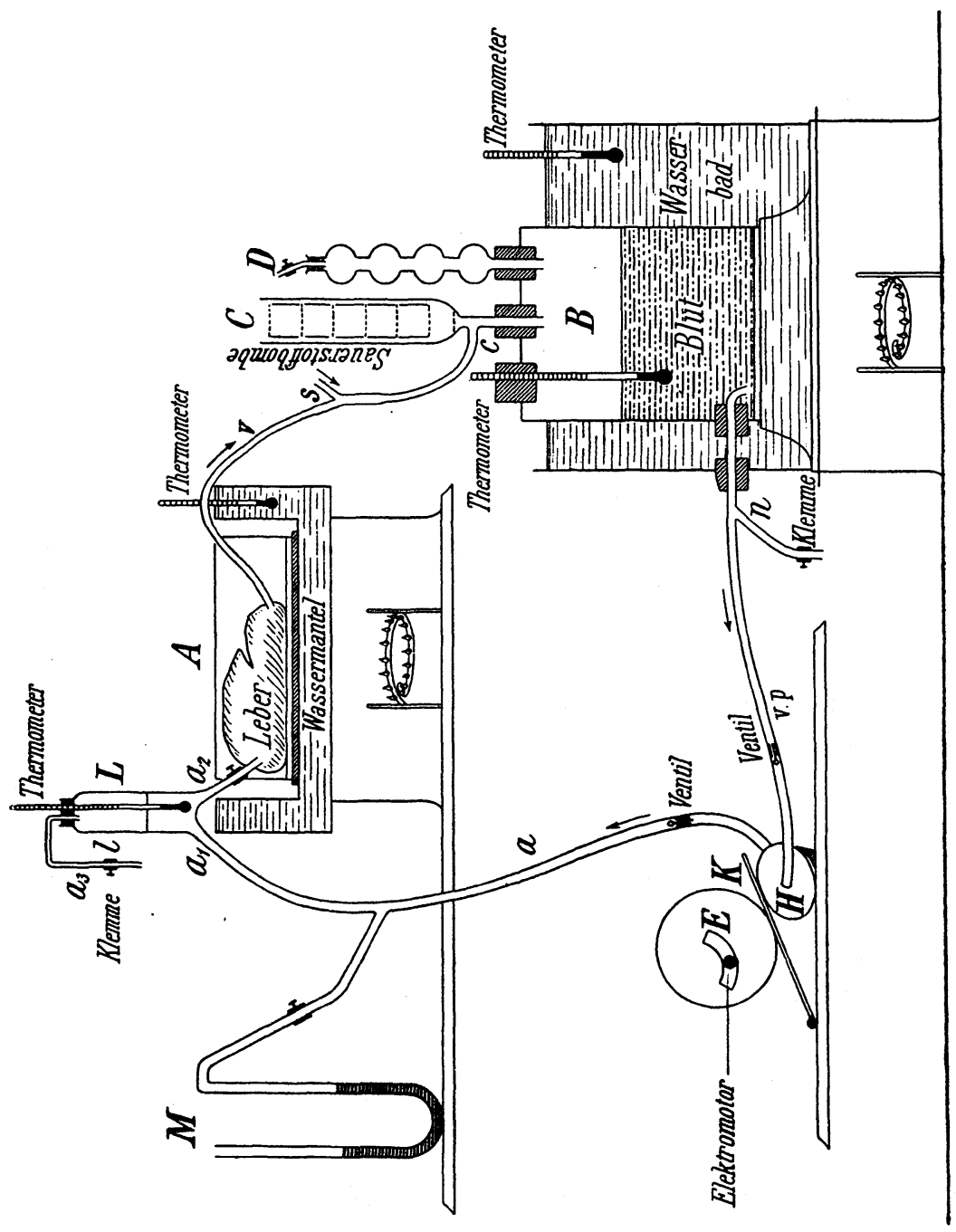

angeordnet sind, daß bei Kompression des Gummiballons durch Exzenter und Klappe die Durchströmungsflüssigkeit durch das eine («arterielle») Ventil in das Organ hineingepreßt wird, während beim Nachlassen der Kompression durch das andere («venöse») Ventil Blut aus einem Vorratsgefäß (B, s. unten) in den Gummiballon einströmt. 
Durch verschiedene Einstellung der exzentrischen Scheibe läßt sich die Kompression des Ballons $\mathrm{H}$ und damit das «Schlagvolumen», durch Veränderung der Umdrehungsgeschwindigkeit des Motors die «Pulsfrequenz» regulieren.

Das zu durchblutende Organ liegt in einer Glasschale, die in einem auf Körpertemperatur eingestellten Thermostaten A (als solcher wurde ein «Sterilisator für Blutserum nach Koch» verwendet) sich befindet. Der Thermostat ist oben durch eine Glasplatte mit 2 Löchern (für die $\mathrm{Zu}$ - und Ableitung des Blutes), nach Bedarf auch noch mit einer Filzplatte abgedeckt.

Zwischen Organ und Herz ist ein einfaches Quecksilbermanometer (M) eingeschaltet, ferner unmittelbar vor dem Organ ein Luftfänger (L), der dazu dient, etwa in das System eingedrungene Luftblasen abzufangen und so Luftembolien $\mathrm{zu}$ vermeiden. Er besteht aus einer ca. $15 \mathrm{~cm}$ langen, $2 \mathrm{~cm}$ weiten Glasröhre, die unten in 2 steil nach abwärts gerichtete Schenkel ausläuft; der eine dieser Schenkel $\left(a_{1}\right)$ steht durch den Gummischlauch a mit dem Herzen in Verbindung und erhält auf diesem Wege Blut; der andere Schenkel $\left(a_{2}\right)$ führt den Blutstrom durch einen Gummischlauch mit Klemme und Kanüle weiter in das Organ. Oben ist der Luftfänger mit einem doppelt durchbohrten Gummischlauch geschlossen; durch die eine Bohrung ist ein Thermometer bis ganz nahe auf den Boden der Glasröhre geführt und gestattet so, die Temperatur des Blutes unmittelbar vor dem Einströmen in das Organ zu kontrollieren, durch die zweite Bohrung geht eine zweimal rechtwinklig gebogene Glasröhre mit Gummischlauch und Klemme $\left(a_{3}\right)$, die dazu dient, die durch den Luftfänger abgefangene Luft nach außen abzulassen. (Während der Durchströmung ist diese Klemme geschlossen; sind Luftblasen in den Luftfänger eingetreten, so können sie abgelassen werden, indem man den Motor abstellt, die Klemme des Schlauches $a_{2}$. schließt, $a_{3}$ öffnet und nun durch vorsichtige Kompression des «Herzens» mit der Hand die Luft durch $a_{3}$ heraustreibt; nach Schließen von $a_{3}$ und Öffnen von $a_{2}$ kann dann die Durchströmung ihren Fortgang nehmen.)

Thermostat mit Organ, Manometer und der an einem Stativ angebrachte Luftfänger stehen auf einem ca. $1 \mathrm{~m}$ hohen Tische. 
Das aus dem Organ abfließende Blut fließt der Schwere folgend durch eine Kanüle und den Schlauch $v$ in das SammelgefäßB. Als Sammelgefäß dient eine ca. 21 fassende W o ulff sche Flasche mit einem seitlich knapp über dem Boden angebrachten und 3 oberen Tubussen.

Sie steht in einem großen Blechtopf, der als Wasserbad $\left(40^{\circ}\right)$ dient, und der ebenfalls einen seitlichen Tubus hat, der dem der Woulffschen Flasche entspricht; in beiden Tuben stecken Gummistopfen, welche eine Glasröhre aufnehmen, die durch einen anschließenden mit einer Klemme armierten Schlauch (vp) das Abfließen des Blutes in das Herz vermittelt; eine Abzweigung mit Klemme ( $\mathrm{n}$ ) gestattet jederzeit Blutproben $\mathrm{zu}$ entnehmen.

Von den oberen 3 Tubussen der W oulff schen Flasche dient der eine zum Einstecken eines Thermometers, die beiden anderen für die beiden Schaumfänger $C$ und $D$. Der Schaumfänger $C$ dient gleichzeitig zur Vervollkommnung der Arterialisierung; er besteht aus einer ca. $35 \mathrm{~cm}$ langen, $5 \mathrm{~cm}$ weiten Glasröhre, in welcher mehrere siebartig durchbrochene Porzellanplatten (als «Filtrierplatten nach Witt» käuflich) durch kurze Glasröhrenstücke über einander aufgestellt sind. Unten ist die Glasröhre stark verjüngt und steckt in dem 2. Tubus der Woulffschen Flasche; durch einen unmittelbar oberhalb der Verjüngung angebrachten seitlichen Ansatz strömt das Blut aus dem Organ ein; durch ein einmündendes Glasrohr (s) ist diesem Blut aus einer Sauerstoff bombe mit Reduzierventil ein genau regulierbarer Strom von Sauerstoff zugeführt worden; von der Einmündungsstelle dieses Glasrohres bis c fließt das Blut mit 0-Blasen gemischt und wird dadurch arterialisiert; die gebildeten Schaumblasen steigen durch die Porzellanplatten des Schaumfängers, die zweckmäßig mit etwas flüssigem Paraffin eingefettet sind, in die Höhe und werden so gebrochen. Weil aber dieser Schaumfänger bei reichlichem Zuströmen von Sauerstoff nicht immer genügt, so ist auf den dritten Tubus der Woulf schen Flasche ein zweiter, einfacher gebauter Schaumfänger (D) aufgesetzt, eine Röhre mit kugelförmigen Erweiterungen, die oben durch einen Gummistopfen mit Glasröhre, 
Schlauch und Klemme armiert ist; durch Regulierung der Klemme kann man einen mehr weniger großen Teil der entweichenden Luft auch hier austreten lassen und so den Schaumfänger G entlasten.

Die Versuche gestalteten sich kurz folgendermaßen: Der Hund wurde durch subcutane Morphiuminjektion betäubt, eine genügende Blutmenge aus der art. carotis oder femoralis entnommen, das Blut durch Schlagen und Durchgießen durch Gazestoff defibriniert und im Verhältnis 1:2 mit Ringerscher Lösung und der Lösung der zu prüfenden Substanz verdünnt; von dieser Durchströmungsflüssigkeit wurde etwas mehr als das Dreifache des Lebergewichtes abgemessen und damit der Apparat gefüllt. Mittlerweile war die Leber aus dem Tier rasch herausgenommen und ihr Gewicht bestimmt worden; in ihre Gefäße (v. portae und v. cava) wurden Glaskanülen eingebunden, das untere Ende der v. cava und das oment. minus durch Ligaturen abgebunden, und das Organ in den künstlichen Kreislauf eingeschaltet; nachdem die Zirkulation einige Minuten in Gang gewesen war, wurde der Überschuß an Durchströmungsflüssigkeit (über das Dreifache des Lebergewichtes hinaus) durch den Schlauch $\mathrm{n}$ entnommen; er diente zur Bestimmung des Acetongehaltes vor der Durchströmung. Wir ließen dieses Kontrollblut absichtlich erst einige Male die Leber passieren, damit eine gleichmäßige Mischung mit dem in den Lebergefäßen zurückgebliebenen Blut erreicht wurde. Es gelingt nicht schwer, so rasch zu arbeiten, daß ungefähr 10 Minuten nach dem Tode des Tieres die Durchströmung beginnt. Von großem Vorteil erweist sich dabei der Luftfänger, der erlaubt, die Luft enthaltende arterielle Kanüle sofort mit dem Schlauch zu verbinden; die Luftblasen steigen dann in dem steilen Schenkel $a_{2}$ rückläufig nach oben. Die Durchströmungen wurden genau 90 Minuten lang fortgeführt und verliefen, von Unterschieden in der Vollkommenheit der Arterialisation abgesehen, alle sehr gleichmäßig und ohne Störung. Vor allem beobachteten wir nie Schwierigkeiten durch Ansteigen des Widerstandes in dem Organe, was wohl auf Verwendung des artgleichen Blutes zurückzuführen ist. Das hellrot einströmende Blut verließ die Leber 
immer tief dunkelrot, obwohl mehrmals vorgenommene Bestimmungen zeigten, daß auf $100 \mathrm{~g}$ Leber berechnet durchschnittlich $75 \mathrm{ccm}$ Blut in der Minute aus der Leber ausströmten, was den Verhältnissen im lebenden Organismus etwa entsprechen dürfte. ${ }^{1}$ )

Bei den Acetonbestimmungen hielten wir uns an die Angaben von Embden. Mit Rücksicht auf die Verdünnung des Blutes mit Ringerlösung wurde 1 Vol. Durchströmungsflüssigkeit mit 1/2 Vol. $3 \%$ iger Salzsäure und 1/2 Vol. konzentrierter $\mathrm{HgCl}_{2}$-Lösung gefällt, filtriert und ein aliquoter Teil des Filtrates destilliert; in dem Destillat bestimmten wir das Aceton nach Messinger-Huppert. Es wurden immer Doppelbestimmungen gemacht. Auf getrennte Aceton- und Acetessigsäurebestimmungen haben wir verzichtet, da bei unserer Fragestellung nur die Gesamtmenge der neugebildeten Substanz von Bedeutung war; deshalb ist oben auch immer nur kurz von *Aceton» die Rede.

Die Darstellung der verwendeten p-Oxyphenylbrenztraubensäure erfolgte durch Kondensation von p-Oxybenzaldehyd mit Hippursäure und Spaltung des Kondensationsproduktes; ${ }^{2}$ ) die p-0xyphenyl- $\alpha$-Milchsäure wurde aus der p-Oxyphenylbrenztraubensäure durch Reduktion mit etwas mehr als der berechneten Menge Natriumamalgam in der Kälte, Ansäuern und Extraktion mit Äther gewonnen; für die Versuche wurde ein aus Wasser umkrystallisiertes Präparat verwendet. Schmelzpunkt lufttrocken $115^{\circ}$ (unkorr.), wasserfrei $140^{\circ}$.

Die Resultate der Versuche sind in folgender Tabelle zusammengestellt.

Aus der Tabelle ergibt sich:

1. daß in den Kontrollversuchen - ohne Zusatz einer Substanz - während der 11/2 Stunden währenden Durchblutung

1) S. Salaskin, Diese Zeitschrift, Bd. XXV, S. 460 (1898). Aus den hier angegebenen Werten Becks läßt sich berechnen, daß durch $100 \mathrm{~g}$ Lebersubstanz in 1 Minute $69,5 \mathrm{ccm}$ Pfortaderblut durchströmen.

2) Neubauer, a. a. 0. S. 229. 


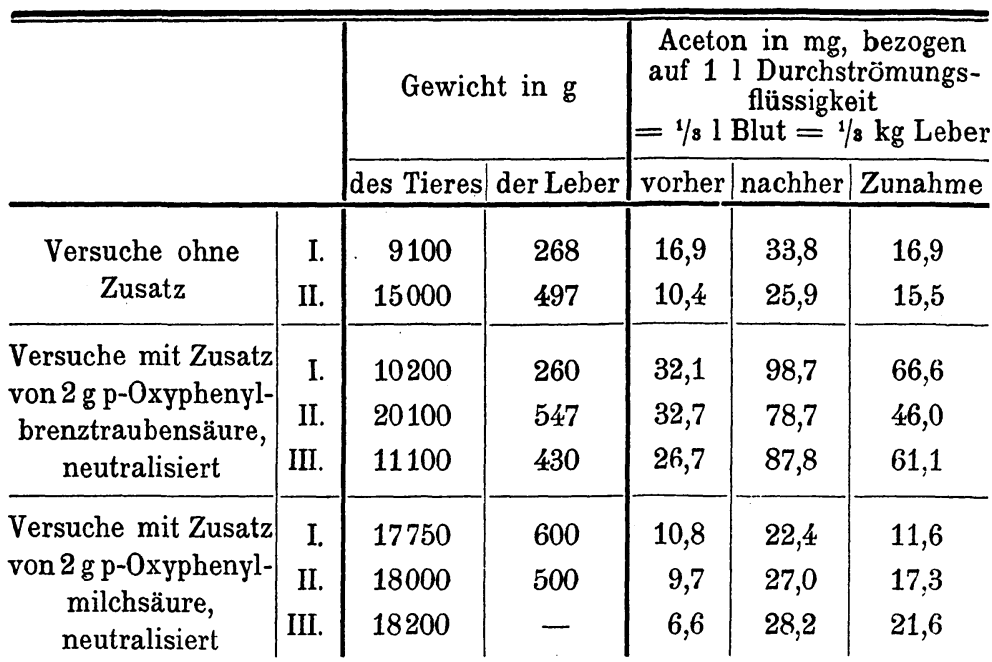

eine Vermehrung des Acetongehaltes der Durchströmungsflüssigkeit eingetreten ist; es entspricht das vollkommen den Erfahrungen von Embden und seinen Mitarbeitern. Die Vermehrung beträgt bei unsern beiden Versuchen im Mittel 16,3 mg für 11 Durchströmungsflüssigkeit resp. ${ }^{1 / 3} \mathrm{~kg}$ Leber, ein Wert, der mit den Resultaten von Embden und Kalberlah ${ }^{1}$ ) sehr gut übereinstimmt.

2. Zusatz von p-Oxyphenylbrenztraubensäure führte in allen 3 Versuchen zu einer beträchtlichen Steigerung dieser Acetonbildung, wenn auch die Werte nicht ganz so hohe sind, wie die von Embden, Salomon und Schmidt nach Zusatz von $2,5-3,0 \mathrm{~g}$ Tyrosin erzielten.

3. Zusatz von p-0xyphenylmilchsäure hat dagegen in 3 einwandfreien Versuchen keine Steigerung dieser Acetonbildung verursacht. ${ }^{2}$ )

1) a. a. 0. S. 6 .

2) Von drei weiteren Versuchen, die mit nicht umkrystallisierten Präparaten von p-0xyphenylmilchsäure angestellt worden waren, hatten allerdings zwei eine gewisse Vermehrung der Acetonbildung ergeben $(42,2,18,5,32,3 \mathrm{mg}$ Aceton pro 11 Durchströmungsflüssigkeit $=1 / \mathrm{s} \mathrm{kg}$ Leber); jedoch sind diese Resultate wahrscheinlich auf eine Verunreinigung der verwendeten Säure mit dem Ausgangsmaterial der Darstellung, der p-Oxyphenylbrenztraubensäure, zurückzuführen. 
Die Versuche ergeben also auch für die Abkömmlinge des Tyrosins einen vollkommenen Parallelismus zwischen dem Schicksal beim Alkaptonuriker und dem Verhalten in der überlebenden Hundeleber: die Substanzen, welche Alkaptonbildner sind (Tyrosin, p-Oxyphenylbrenztraubensäure) liefern in der Hundeleber Aceton; die p-Oxyphenylmilchsäure, die nicht in Alkapton übergeht, führt auch zu keiner Acetonvermehrung.

Die Untersuchungen bestätigen ferner die durch die Alkaptonversuche gewonnene Anschauung, daß der Abbau des Tyrosins nicht über die p-Oxyphenylmilchsäure führen kann, sehr wohl aber über die p-Oxyphenylbrenztraubensäure; sie rechtfertigen damit die Übertragung dieser Schlußfolgerung auch auf den normalen Organismus.

Bemerkenswert bleibt der Unterschied in dem Verhalten der Abkömmlinge der beiden aromatischen Aminosäuren: die Alkoholsäure, die sich vom Phenylalanin ableitet, ist Alkaptonbildner und Acetonbildner, die des Tyrosins ist keines von beiden; die Ketonsäuren beider bilden Alkapton, und - wenigstens die vom Tyrosin sich ableitende - auch Aceton (die Ketonsäure des Phenylalanins ist im Durchblutungsversuch noch nicht geprüft, doch dürfte sie sich dabei aller Voraussicht nach ebenfalls als Acetonbildner erweisen). 